\title{
Évaluation du facteur de mise en suspension de contamination dans les installations nucléaires
}

\author{
D. BOULAUD ${ }^{1}$, P. GÉRASIMO ${ }^{2}$, G. MARTIN ${ }^{3}$, L. MARTINOT $^{4}$, \\ A. NOURREDDINE ${ }^{5, *}$, J.C. SENS ${ }^{5}$
}

(Manuscrit regu le 15 novembre 2002, accepté le 28 mai 2003 )

RÉSUMÉ Ce travail entre dans le cadre du dossier «maîtrise du risque alpha sur des installations nucléaires » qui est issu du projet propreté radiologique d'EDF. Une validation du facteur réaliste de mise en suspension de contamination est nécessaire afin de s'assurer que les dispositions prises par EDF dans le cadre de la radioprotection des travailleurs lors des interventions sur des chantiers à risque alpha sont adaptées. Une expérimentation du facteur de mise en suspension sur l'arrêt de tranche 3 de la centrale nucléaire Cattenom, qui a subit une dissémination de matière fissile pendant l'arrêt de tranche en janvier 2001 a été réalisée. Le bilan des activités surfaciques et atmosphériques sur trois types de chantiers (pressuriseur, générateur de vapeur et piscine du bâtiment réacteur) est analysé. L'ensemble des valeurs obtenues permet de fixer et d'adopter en radioprotection opérationnelle la valeur la plus probable du facteur de mise en suspension des particules émettrices $\alpha F_{\text {MFS }}=1 \times 10^{-6} \mathrm{~m}^{-1}$.

ABSTRACT Evaluation of the resuspension factor in nuclear power installations.

This work is part of the EDF project "control of $\alpha$-particle radiation risk in nuclear power stations". A realistic determination of the resuspension factor of contamination is necessary to be certain that the health protection measures taken by the EDF for their on-site workers are applicable. An experimental determination of the resuspension factor during the programmed maintenance of Unit 3 of the Cattenom power station, which suffered a dispersion of fissile material during a stoppage in January 2001, has been performed. The values of the surface and atmospheric activity concentrations at three workspace (pressure unit, steam generator and pool of the reactor building) are analysed. The results allow setting the most realistic value of the resuspension factor for $\alpha$-particle for operational radiation protection at $F_{M E S}=1 \times 10^{-6} \mathrm{~m}^{-1}$.

\section{Introduction}

Dans le domaine de la radioprotection, la maîtrise de la radioactivité de l'atmosphère ambiante est un paramètre essentiel pour assurer la sécurité des

\footnotetext{
IRSN, DPEA/SERAC, bâtiment 389, B.P. 68, 91192 Gif-sur-Yvelle Cedex, France.

Service de prosection radiologique des anmés, I bis ruc du licutenant Raoul Batany, 92141 Clamart, France.

CEA, DAM, B.P. 12, 91680 Bıuyc̀res-le-Châted, France.

4 Laboratoire de Radiochimie, Université de Liègc, bâtiment B16, Sart-Tilman, 40000 Liège. Belgique.

5 Institut de Recherches Subatomiques. 23 rue de Loess, B.P. 28. 67037 Strasbourg Cedex 2. France.

* Auteur pour la correspondance.
} 
intervenants qui travaillent sur un site nucléaire. En pratique, la contamination de l'air dans une enceinte fermée est le résultat d'une contamination surfacique initiale non fixée. Si la mesure d'une contamination labile au niveau du sol est aisée et rapide, il n'en est pas de même pour une mesure volumique alors que c'est la connaissance de ce paramètre qui amène à imposer directement les précautions à prendre par le personnel qui opère sur le terrain ou sera amené à le faire.

Le lien entre contamination surfacique et contamination volumique de l'air n'est pas caractérisable par un rapport bien défini, généralisable à toutes situations, car la mise en suspension dans l'atmosphère de particules déposées sur le sol dépend de divers paramètres : la taille et la densité des matériaux contaminants, leur composition chimique, les déplacements d'air provoqués par la ventilation, les mouvements des personnes présentes ainsi que la nature des travaux effectués.

Une approche quantifiée globale de l'ensemble de ces paramètres a conduit à la définition d'un facteur de mise en suspension, $F_{M E S}$, égal au quotient de l'activité volumique par l'activité surfacique qui lui a donné naissance.

\section{Point bibliographique sur la mise en suspension de particules}

La caractérisation de la mise en suspension, par écoulement d'air, de particules déposées sur une surface, a suscité et suscite encore un grand nombre d'études théoriques et expérimentales. Si la connaissance théorique du phénomène est relativement claire dans le cas d'une particule sphérique isolée sur une surface plane dans un écoulement d'air turbulent, il existe encore, en revanche, de nombreuses zones d'ombre lorsque l'on prend en compte des paramètres relatifs à la contamination déposée (monocouche, multicouche, amas), à l'ensemble des particules qui constituent la contamination (distribution en dimensions, propriétés physico-chimiques), à la surface (rugosité, élasticité, état électrostatique), à l'écoulement d'air (accélération, niveau de turbulence) ou encore aux paramètres environnementaux (humidité relative, température) (Witschger, 1999).

\section{1. Études théoriques}

Il existe dans la littérature spécialisée des synthèses récentes dédiées à l'analyse des travaux théoriques sur la mise en suspension par écoulement d'air (Ziskind et al., 1995 ; Alloul-Marmor, 2002). Ces travaux considèrent exclusivement le comportement à proximité immédiate de la paroi où la particule est déposée, c'est-à-dire dans la couche limite.

Deux types de modèles de mise en suspension (détachement de la surface) existent, fondés sur deux approches différentes :

- un bilan entre les forces d'adhérence de la particule sur la surface et les forces 
aérodynamiques liées à l'écoulement (Corino et Brodkey, 1969 ; Cleaver et Yates, 1973),

- un bilan entre l'énergie transférée de l'écoulement vers la particule et la barrière de potentiel d'adhérence (Reeks et al., 1988).

Les travaux de ces différents auteurs ont permis une avancée remarquable dans la compréhension des mécanismes de mise en suspension d'une particule. En effet, d'un soulèvement très simpliste de la particule basé sur un équilibre des forces ou une accumulation d'énergie par la particule, ces auteurs ont su faire évoluer les développements théoriques en s'attachant aux déplacements infinitésimaux de la particule avant son envol dans l'écoulement libre. C'est le cas notamment des travaux de Reeks et Hall (2001) qui intègrent les moments des forces dans le mécanisme de détachement en combinant les deux approches décrites précédemment (bilan des forces et bilan d'énergie). Ce modèle, amélioré récemment par Biasi et al. (2001), paraît être le plus achevé permettant de décrire au mieux les travaux expérimentaux existants. Cependant, il n'est strictement valable que dans le cas de la mise en suspension d'une particule sphérique isolée sur une surface.

\section{2. Études expérimentales}

Dans la littérature spécialisée se trouvent des études expérimentales sur le thème général de la mise en suspension de particules par écoulement d'air. Sehmel (1980) et, plus tard, Nicholson (1988) présentent des synthèses bibliographiques décrivant plus particulièrement des études expérimentales dans le domaine de l'environnement. Ils constatent, en fonction des différentes situations, que le facteur de mise en suspension $\left(\mathrm{F}_{\mathrm{MES}}\right)$ peut être compris entre $1 \times 10^{-10}$ et $1 \times 10^{-2} \mathrm{~m}^{-1}$.

Dans le domaine de la radioprotection, Brodsky (1980) fait un constat analogue en citant des valeurs de $\mathrm{F}_{\mathrm{MES}}$ variant de $1 \times 10^{-13}$ à $1 \times 10^{-3} \mathrm{~m}^{-1}$. Les premières estimations expérimentales de ce facteur, concernant des laboratoires de radiochimie, ont conduit à une valeur moyenne $F_{M E S}=1 \times 10^{-6} \mathrm{~m}^{-1}$. D'autres mesures menées à l'extérieur du laboratoire conduisent à la même valeur et il en résulte qu'un facteur $F_{M E S}$ égal à $1 \times 10^{-6} \mathrm{~m}^{-1}$ a été recommandé dans des conditions usuelles de travail bien que ce facteur puisse atteindre $1 \times 10^{-5} \mathrm{~m}^{-1}$ lors d'activités soutenues. On peut également citer le rapport publié par le DOE (Department of Energy) des États-Unis en 1994 (DOE handbook, 1994).

Très récemment Alloul-Marmor (2002) a synthétisé plus particulièrement les études expérimentales en laboratoire destinées à la validation des modèles décrits ci-dessus. À partir d'une vingtaine de travaux, elle constate :

- que la validation des modèles est correcte mais souvent limitée à des conditions expérimentales très particulières, 
- que les études expérimentales sont difficiles à comparer tant les conditions de réalisation sont variées,

- qu'il est difficile d'extraire de ces études des informations en terme de coefficient de mise en suspension ( $\mathrm{K}_{\mathrm{MES}}$ ou $\mathrm{F}_{\mathrm{MES}}$, voir paragraphe 3) qui puissent être directement utilisables dans les analyses de sûreté des installations ou en radioprotection opérationnelle.

À partir de l'ensemble des travaux disponibles, force est de constater qu'il est difficile de retenir une valeur du facteur de mise en suspension unique, généralisable à toutes les situations.

\section{Définition des coefficients de mise en suspension}

On distingue différents coefficients qui permettent de caractériser la mise en suspension de contamination. Il est donc important de les définir précisément.

\subsection{Fraction mise en suspension ( $\left.\mathrm{K}_{\mathrm{MES}}\right)$}

Elle est définie par le rapport de la quantité totale de matériaux contaminants mise en suspension sur la quantité initiale de matériau présente dans un volume ou sur une surface. Il s'exprime donc sans unité :

$$
\mathrm{K}_{\mathrm{MES}}=\frac{\text { quantité mise en suspension }}{\text { quantité initiale }} .
$$

Ce coefficient est utile lorsqu'il s'agit de quantifier la mise en suspension de particules provenant d'objet massif (tas de poudre à titre d'exemple).

\subsection{Facteur de mise en suspension ( $\left.\mathrm{F}_{\mathrm{MES}}\right)$}

Il est défini par le rapport de la concentration volumique de la contamination présente dans le fluide porteur sur la concentration surfacique de cette contamination :

$$
\mathrm{F}_{\mathrm{MES}}=\frac{\text { concentration volumique }}{\text { concentration surfacique }} \quad \text { unité }: \mathrm{l}^{-1} \text {. }
$$

Ce coefficient est assez répandu dans la littérature. Il est notamment utilisé pour quantifier la mise en suspension dans l'environnement extérieur, par exemple pour décrire le transport éolien de poussières.

On peut noter que dans le cas d'un local sans échange avec l'extérieur, de volume $\mathrm{V}$ et de surface contaminée $\mathrm{S}$, en supposant que la concentration 
volumique de la contamination en suspension et le dépôt surfacique sont homogènes, les coefficients $\mathrm{F}_{\mathrm{MES}}$ et $\mathrm{K}_{\mathrm{MES}}$ sont reliés par l'expression suivante :

$$
\mathrm{K}_{\mathrm{MES}}=\frac{\mathrm{V}}{\mathrm{S}} \mathrm{F}_{\mathrm{MES}}
$$

\subsection{Taux de mise en suspension ( $\mathrm{T}_{\mathrm{MES}}$ )}

Il est défini par le rapport du débit d'émission de la contamination sur la quantité initiale présente :

$$
\mathrm{T}_{\text {MES }}=\frac{\text { débit d'émission }}{\text { quantité initiale }} \quad \text { unité }: \mathrm{T}^{-1} \text {. }
$$

\subsection{Flux de mise en suspension ( $\phi_{\mathrm{MES}}$ )}

Il est défini par la masse de contaminants mise en suspension par unité de temps et par unité de surface :

$$
\Phi_{\mathrm{MES}}=\frac{\text { débit massique d'émission }}{\text { surface contaminée }} \quad \text { unité : } \mathrm{M}^{-2} \mathrm{~T}^{-1} \text {. }
$$

Le choix d'un coefficient repose souvent sur les grandeurs mesurées lors des études. Dans notre cas, le choix s'est porté naturellement sur le facteur de mise en suspension sachant que les grandeurs mesurées sont les concentrations volumique et surfacique de la contamination.

\section{Projet EDF : maîtrise du risque $\alpha$ sur les chantiers}

Électricité de France (EDF) a mis en place en 1999 un projet «Propreté radiologique ». Ce projet englobe les thématiques suivantes : le combustible nucléaire, la maîtrise du risque alpha, les rejets, les déchets, les transports, ... Parmi les problèmes identifiés, la surveillance de l'activité $\alpha$ et la maîtrise de ce risque sur les installations, notamment lors des arrêts de tranches des centrales nucléaires, a été particulièrement étudiée.

La maîtrise du risque $\alpha$ repose sur l'identification et la quantification de la contamination dont découlent les dispositions appropriées de protection des intervenants. Les modalités des mesures $\alpha$ effectuées, la discussion de leur validité et l'adoption d'un facteur réaliste de mise en suspension des particules ont fait l'objet d'une mission d'un comité scientifique d'experts indépendants (IRSN, SPRA, CEA/DAM, CNRS/RReS, Université de Liège). Ce comité a participé à la définition et la validation des mesures demandées par EDF au travers un cahier des charges précis et effectuées sous la responsabilité du Laboratoire de transfert de contamination du CEA Cadarache. Il a interprété l'ensemble des données 
recueillies lors des différents campagnes de mesures réalisées lors de l'arrêt de tranche 3 de la centrale nucléaire de Cattenom. Il s'agit d'une démarche complexe basée sur un cahier des charges imposant des mesures radiologiques à différents points-clefs : le trou d'homme du pressuriseur (THPZR), le générateur de vapeur (GV4) au niveau de la boîte à eau et des presse-joints (en phase humide puis en phase sèche), la piscine du bâtiment réacteur après vidange mais avant décontamination, à la fois sur le fond et sur les parois. De l'ensemble de ces mesures, une valeur réaliste et représentative de $F_{\text {MES }}$ sera dégagée pour caractériser les chantiers à risque $\alpha$, mais les émetteurs $\beta$ très énergétiques ( $\mathrm{E}>$ $320 \mathrm{keV}$ ) seront également pris en considération.

\section{Matériels et méthodes}

Le facteur de mise en suspension $F_{\text {MES }}$ est défini par le rapport de l'activité volumique à proximité d'une contamination surfacique sur l'activité surfacique source de l'activité volumique. Dans cette étude, l'activité volumique a été mesurée par prélèvement d'aérosols tandis que l'activité surfacique a été mesurée par frottis.

\subsection{Mesure de la contamination surfacique}

La procédure de prélèvement est basée sur l'utilisation d'un frottis normalisé (Décret, 2003), frotté de façon régulière sur une surface de $10 \mathrm{~cm} \times 10 \mathrm{~cm}$. Le frottis est ensuite transféré, face frottée visible, dans une boîte de Pétri, préalablement étiquetée sur le fond. L'étiquette porte les informations suivantes : référence du prélèvement, lieu, date, heure. La boîte de Pétri est fermée hermétiquement. Pour chaque frottis, deux mesures sont réalisées :

- une mesure de radioprotection sur le site de la centrale permet d'estimer l'activité $\alpha$ et $\beta$ totale. Cette mesure est réalisée à l'aide d'un contaminamètre ECM21 muni d'une sonde SBM pour les rayonnements $\beta$ (sensibilité $1 \mathrm{~Bq} / \mathrm{cm}^{2}$ ) et d'une sonde SMIA pour la mesure $\alpha$ (sensibilité $1 \mathrm{~Bq} / \mathrm{cm}^{2}$ ). La mesure est réalisée dans le laboratoire du bâtiment des auxiliaires nucléaires afin de s'affranchir du bruit de fond ambiant ;

- une mesure de laboratoire par spectométrie $\alpha$ en chambre à grille sur l'ensemble des frottis, complétée par des mesures du ${ }^{90} \mathrm{Sr}$ en scintillation liquide et des émetteurs gamma par spectrométrie (germanium HP). Ces mesures sont effectuées dans le laboratoire d'analyse du CEA de Cadarache.

Pour remonter à l'activité surfacique source, le rendement du frottis est pris égal à $10 \%$ selon les directives de la norme NF-ISO 7503-1 (1998) relative à l'évaluation de la contamination de surface. 


\subsection{Mesure de l'activité volumique}

L'activité volumique est déterminée au voisinage immédiat de la contamination surfacique par prélèvements d'aérosols. Deux modes de prélèvements ont été réalisés.

(1) Un prélèvement de 10 minutes par aspirateur de type Staplex/Radeco, à un débit de $1 \mathrm{~m}^{3} / \mathrm{min}$, a été pratiqué sur les trois types de chantier. Les filtres utilisés sont fabriqués par SOFILTRA (référence 3052/11). Ce sont des filtres de diamètre $105 \mathrm{~mm}$, en fibre végétale jaune $\left(100 \mathrm{~g} / \mathrm{m}^{2}\right)$; l'efficacité mesurée avec un aérosol de DOP (dioctylphtalate monodispersé de diamètre $0,3 \mu \mathrm{m}$ ) est de $95 \%$ et la perte de charge est de $70 \mathrm{~Pa}$ (mesures à $\mathrm{l} \mathrm{cm} / \mathrm{s}$ ). Les filtres sont placés verticalement à l'entrée du dispositif d'aspiration.

Le choix de la durée de l'échantillonnage résulte d'un compromis entre un volume d'air maximal requis pour une bonne analyse statistique et un temps de présence minimal sur le chantier imposé pour la protection des intervenants. Le cahier des charges de l'EDF exige le prélèvement d'une activité égale à 5 fois la limite de détection par spectrométrie $\alpha$, soit environ $0,01 \mathrm{~Bq}$. L'activité volumique prélevée tient compte de la limite dérivée de concentration dans l'air (LDCA) des principaux isotopes, particulièrement le ${ }^{239} \mathrm{Pu}$.

(2) Le second type de prélèvement impliquant un impacteur, basé sur la classification inertielle de particules, a été effectué sur le chantier générateur de vapeur en phase sèche (chantier GV4).

Le principe de classification inertielle de particules en suspension dans un gaz dépend d'une accélération rapide du gaz vecteur, celle ci pouvant être mise en ouvre, notamment, par déviation de la direction de l'écoulement. Dans ce cas, les particules les plus grosses ayant suffisamment d'inertie traverseront les lignes de courant du gaz porteur et s'échapperont de l'écoulement et pourront être collectées, alors que les particules plus fines resteront dans cet écoulement.

Dans les impacteurs cette classification inertielle est mise en œuvre en dirigeant un jet d'air à haute vitesse contre une surface solide appelée plaque de collecte. La déviation du jet d'air au voisinage de la surface va provoquer l'impact des particules de tailles supérieures à un certain diamètre dit de "coupure » alors que les particules plus fines resteront en suspension dans le gaz. Le nombre de Stokes de la particule est le paramètre sans dimension qui permet de prévoir si une particule impactera ou non sur la plaque de collecte. Il est défini par :

$$
\mathrm{S}_{\mathrm{tk}}=\tau_{\mathrm{P}} \frac{\mathrm{V}}{\mathrm{R}}
$$


où $\tau_{p}$ représente le temps de relaxation de la particule, grandeur caractéristique de l'inertie, $\mathrm{V}$ la vitesse moyenne du gaz et $\mathrm{R}$ une dimension caractéristique du système qui peut être le rayon du conduit d'accélération du fluide porteur (Renoux et Boulaud, 1998). Rappelons que le terme «temps de relaxation » caractérise le temps mis par une particule pour ajuster sa vitesse à une nouvelle condition de champs de force. Le nombre de Stokes ne permet pas de décrire les effets parasites tels que les pertes sur les parois, les rebonds, le ré-entraînement, mais il prédit précisément si la particule se déposera ou non sur la plaque de collecte. Physiquement, le nombre de Stokes représente le rapport entre le temps de relaxation de la particule et le temps de séjour du fluide le long de la plaque de collecte. Les étages d'impacteur avec des géométries similaires mais des dimensions ou des vitesses variables aboutissent à des efficacités de collecte communes lorsqu'elles sont exprimées en fonction du nombre de Stokes.

Le nombre de Stokes correspondant à une efficacité de collecte de $50 \%$ a une valeur comprise entre 0,22 et 0,25 pour la plupart des impacteurs à jets circulaires. Pour collecter des particules de plus en plus petites, ayant donc des temps de relaxation de plus en plus faibles, on comprend bien qu'il est nécessaire d'augmenter $\mathrm{V}$ et/ou de diminuer R. En disposant plusieurs étages en série avec des dimensions de plus en plus petites et des vitesses de plus en plus grandes, on peut ainsi fractionner un aérosol polydispersé en plusieurs classes.

La masse ou la radioactivité, recueillie sur chaque plaque de collecte correspondant aux différents étages, peut être mesurée et permettre l'établissement de la granulométrie en masse ou en activité de l'aérosol.

Dans notre étude, l'impacteur choisi est le Dekati PM10 (ECOMESURE, France). Cet appareil dispose de 3 étages avec des diamètres de coupure de $10 \mu \mathrm{m}$, $2,5 \mu \mathrm{m}$ et $1 \mu \mathrm{m}$, plus un étage de filtration qui recueille les particules submicroniques. Ce faible nombre d'étages induit une résolution en taille relativement médiocre, mais présente l'avantage, dans le cas des mesures radioactives, de ne pas disperser l'activité sur un trop grand nombre d'étages avec le risque de se retrouver pour chaque étage sous la limite de détection. Dans les conditions ambiantes, le débit de prélèvement est de $9,8 \mathrm{l} / \mathrm{min}$ ce qui implique des durées d'échantillonnage importantes (environ 13 heures).

Les fenêtres «temps » des deux méthodes de prélèvement, par l'aspirateur Radeco et l'impacteur Dékati, sont tout à fait différentes. L'impacteur peut intégrer des événements transitoires non répétitifs, tandis que la mesure rapide par aspiration (10 minutes) ne sera pas nécessairement représentative de toutes les situations. L'emploi de l'impacteur correspond mieux à l'évaluation des conditions d'une intervention prolongée - maintenance, décontamination - tandis 
que l'échantillonnage rapide permet d'appréhender le risque encouru lors d'une intervention d'urgence ou limitée dans le temps.

\subsection{Localisation et bilan global des prélèvements}

Les endroits et les modes de prélèvements se présentent comme suit :

- six frottis de $100 \mathrm{~cm}^{2}$ au niveau du pressuriseur et de la tape du pressuriseur,

- quatre frottis $\left(100 \mathrm{~cm}^{2}\right)$ sur la piscine-sol et parois,

- quatre frottis $\left(100 \mathrm{~cm}^{2}\right)$ sur le générateur de vapeur GV4, phase humide puis phase sèche,

- deux frottis $\left(100 \mathrm{~cm}^{2}\right)$ sur le presse-joint, en phase sèche, dont les résultats sont comparés avec les résultats de quatre grattages de $4 \mathrm{~cm}^{2}$ pratiqués au même endroit,

- un prélèvement d'aérosol par aspiration de $1 \mathrm{~m}^{3}$ seulement au trou d'homme du pressuriseur en phase humide et un aérosol de $10 \mathrm{~m}^{3}$ en phase sèche,

- deux aérosols $\left(10 \mathrm{~m}^{3}\right)$ au fond et au bord de la piscine (BR) avec peu d'intervenants dans les circonstances du chantier,

- deux aérosols $\left(10 \mathrm{~m}^{3}\right)$ au générateur $\mathrm{GV4}$, en phase humide et en phase sèche.

L'ensemble de ces mesures coordonnées (contamination surfacique + contamination volumique) est représentatif de la situation des entités concernées lors de l'arrêt de tranche avant décontamination. Les relevés faits avec l'impacteur donnent un jeu plus complet d'informations mais au seul niveau du générateur GV4.

\section{Résultats expérimentaux}

Au total, 30 échantillons ont été analysés ; 29 isotopes $\left({ }^{51} \mathrm{Cr},{ }^{54} \mathrm{Mn},{ }^{56} \mathrm{Ni},{ }^{57} \mathrm{Co}\right.$, ${ }^{58} \mathrm{Co},{ }^{60} \mathrm{Co},{ }^{59} \mathrm{Fe},{ }^{65} \mathrm{Zn},{ }^{90} \mathrm{Sr},{ }^{95} \mathrm{Zr},{ }^{95} \mathrm{Nb},{ }^{99 \mathrm{~m}} \mathrm{Tc},{ }^{103} \mathrm{Ru},{ }^{110 \mathrm{~m}} \mathrm{Ag},{ }^{113} \mathrm{Sn},{ }^{124} \mathrm{Sb}$, ${ }^{125} \mathrm{Sb},{ }^{131} \mathrm{I},{ }^{132} \mathrm{Te},{ }^{140} \mathrm{Ba},{ }^{140} \mathrm{La},{ }^{141} \mathrm{Ce},{ }^{144} \mathrm{Ce},{ }^{239} \mathrm{~Np},{ }^{241} \mathrm{Am},{ }^{242} \mathrm{Cm},{ }^{243} \mathrm{Cm}$, ${ }^{244} \mathrm{Cm},{ }^{238} \mathrm{Pu}$ ) ont été identifiés à chaque fois et quantifiés pour autant qu'une statistique significative de comptage ait pu être dégagée. La présentation détaillée de tous les résultats sort du cadre de cet article mais ils sont accessibles par la note technique DEC/S3C/01-101 du centre CEA de Cadarache (Rabu et al., 2002). À titre d'exemple, le tableau I donne, pour le chantier boîte à eau GV4 en phase humide, les activités surfaciques et atmosphériques correspondantes ainsi que la valeur moyenne du facteur de mise en suspension $\mathrm{F}_{\mathrm{MES}}$ pour chaque isotope. Ces facteurs sont calculés en tenant compte du rendement de frottis pris égal à $10 \%$. Les incertitudes sur les activités surfaciques et atmosphériques correspondent à des intervalles de confiance à $95 \%$. Les valeurs de $\mathrm{F}_{\mathrm{MES}}$ ne sont pas calculées lorsqu'une des deux mesures d'activité n'est pas quantifiable. Dans le tableau II sont résumés les facteurs $F_{\text {MES }}$, pour chaque isotope et pour les trois chantiers : fond de piscine BR, boîte à eau (GV4) après séchage et pressuriseur en phase sèche et humide. 
TABLEAU I

Activités brutes des frottis et prélèvement atmosphérique et facteurs de mise en suspension de la contamination sur le chantier boite à eau GV4 en phase humide. $S_{1}$ : entrée boîte à eau GV4; $S_{2}$ : sortie boîte à eau GV4. F $\mathrm{MEs}$ représente la moyenne des valeurs obtenues à partir de l'activité atmosphérique et des deux activités frottis $S_{1}$ et $S_{2}$.

Total activity of smears, atmospheric sampling and atmospheric resuspension factors of the contamination at the water reservoir work site of the vapor generator GV4 in humid phase. $S_{1}$ : entry of the water reservoir; $S_{2}$ exit of the water reservoir. $F_{M E S}$ represents the average of the values obtained from atmospheric activity and the two activities smear $S_{1}$ and $S_{2}$.

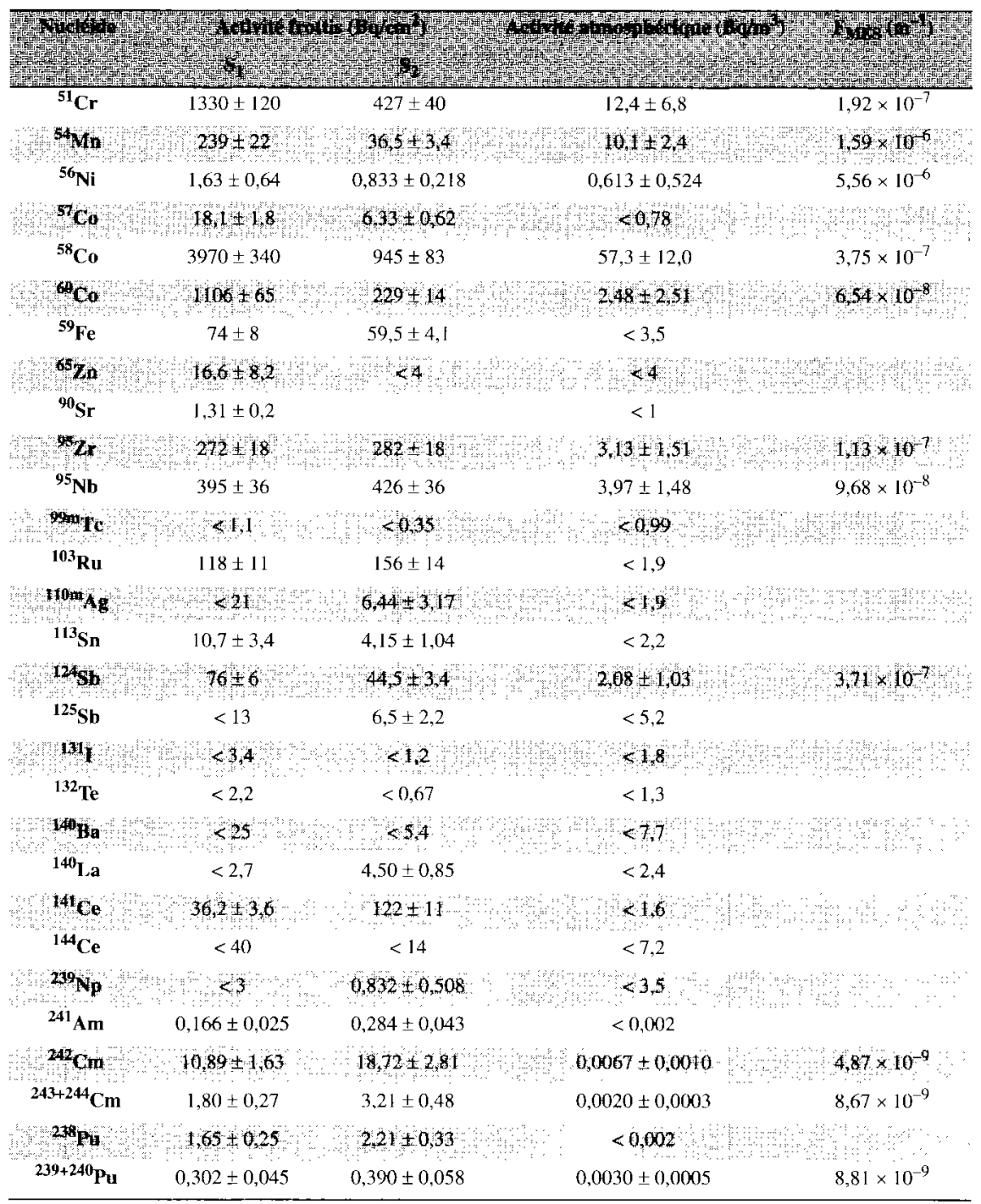




\section{TABLEAU II}

Facteur de mise en suspension exprimé en $\mathrm{m}^{-1}$ de la contamination sur les chantiers fond de piscine BR, boite à eau (GV4) et pressuriseur pour les différents isotopes.

Atmospheric resuspension factor in $\mathrm{m}^{-1}$ for different isotopes contaminations workplaces on the the floor of the BR pool, the water chamber of the vapor generator GV4 and of the pressurizer.

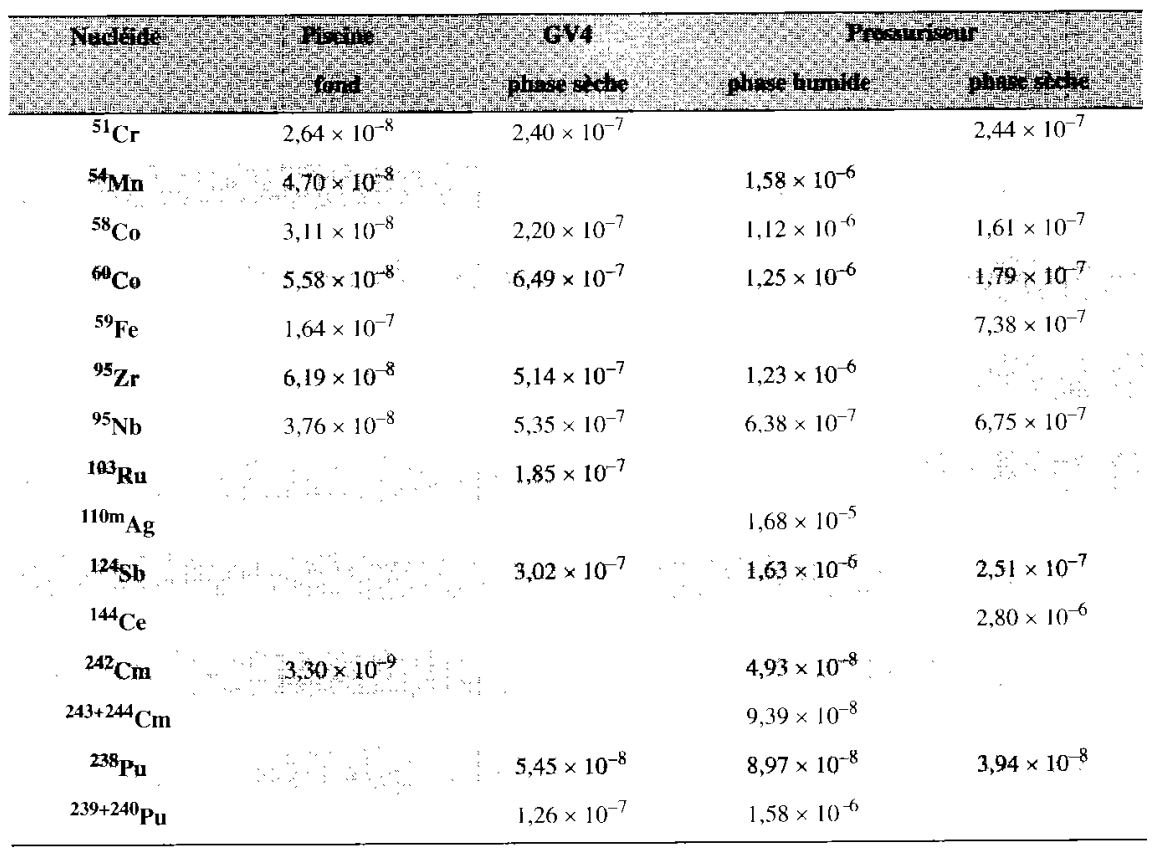

Une attention particulière a été portée aux données fournies par l'impacteur (chantier générateur vapeur, GV4, phase sèche uniquement). Le tableau III montre la répartition granulométrique de l'activité des différents isotopes sur le prélèvement atmosphérique réalisé sur le chantier GV4 phase sèche pour les différents diamètres de coupure de l'impacteur. Le diamètre aérodynamique médian en activité et l'écart type géométrique sont déterminés selon l'ajustement à une droite de Henry. Le diamètre aérodynamique médian en activité est de l'ordre de $1 \mu \mathrm{m}$ quel que soit le nucléide et les écarts types géométriques sont compris entre 1,6 et 8 . Les valeurs élevées des écarts types géométriques observés ne signifient pas que l'écart type géométrique instantané de la distribution en dimensions atteint ces valeurs. Elles sont liées à un prélèvement de très longue durée au cours duquel la dimension moyenne des aérosols peut varier induisant ainsi un «étalement» de la distribution en taille autour de la valeur médiane. Une comparaison graphique des valeurs de $\mathrm{F}_{\mathrm{MES}}$, obtenues en échantillonnage rapide (aspirateur Radéco) et en échantillonnage long (impacteur) est présentée en 


\section{TABLEAU III}

Répartition granulométrique de l'activité atmosphérique $\left(\mathrm{Bq} / \mathrm{m}^{3}\right)$ sur les différents étages de l'impacteur (chantier GV4 phase sèche). DAMA : diamètre aérodynamique médian en activité. ETG : écart type géométrique.

Distribution by particle size of the atmospheric activity in $\mathrm{Bq} / \mathrm{m}^{3}$ at different stages of the impactor (vapor generator work site in the dry phase). DMA: mean aerodynamic diameter for each activity. ETG: geometrical standard deviation.

\begin{tabular}{|c|c|c|c|c|c|c|c|}
\hline 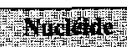 & M P & 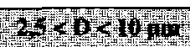 & 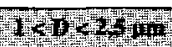 & 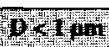 & (bilit & 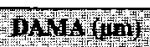 & When \\
\hline & & 11,10 & 12,40 & 33,50 & 57,00 & 1,1 & 1,6 \\
\hline Mn & 2,57 & 4,34 & 5,15 & 6,76 & 18,82 & 17 & 5,0 \\
\hline${ }^{57} \mathrm{Co}$ & 0,73 & 0,43 & 0,57 & 0,58 & 2,31 & 3,4 & 7,9 \\
\hline${ }^{58} \mathrm{Co}$ & 50,10 & 76,80 & 83,50 & 110,00 & 320,40 & 18 & 5,2 \\
\hline${ }^{60} \mathrm{Co}$ & 14,80 & 23,50 & 26,90 & 33,60 & 98,80 & 1,8 & 5.0 \\
\hline${ }^{95} Z_{K}$ & & 1,99 & 2,44 & 4,73 & $9,16:$ & $1 ; 2$ & 1,6 \\
\hline${ }^{95} \mathrm{Nb}$ & 11,30 & 10,80 & 13.20 & 27,30 & 62,60 & 1,3 & 8,9 \\
\hline${ }^{242} \mathrm{Cm}$ & 0,0087 & 0,0697 & 0,103 & 0,79 & 0,360 & 1,0 & 3,2 \\
\hline${ }^{243+244} \mathrm{Cm}$ & 0,00649 & 0,0130 & 0,0130 & 0.0247 & 0,0572 & 1,3 & 5,4 \\
\hline${ }^{238} \mathrm{Pu}$ & 0,00130 & 0,0117 & 0,0143 & 0,0377 & 0,0650 & 0,8 & $3 ; 4$ \\
\hline $239+241 \mathrm{Pu}$ & 0,00519 & 0,00779 & 0,00779 & 0,0130 & 0,0338 & 1,6 & 5,9 \\
\hline
\end{tabular}

figure 1. On note que les valeurs de $\mathrm{F}_{\text {MES }}$ obtenues au moyen de l'impacteur sont les plus cohérentes pour les émetteurs alpha et gamma.

\section{Discussions}

Les activités $\alpha$ analysées dans cette étude sont souvent proches de la limite de détection. Ces résultats traduisent un très faible niveau de contamination lors de l'arrêt de tranche et ils fournissent des repères intéressants qui corroborent d'autres observations dans un domaine où il existe peu de données dans la littérature. Il faut aussi signaler que des différences importantes de contamination apparaissent au niveau du pressuriseur, en passant de la phase humide, où la température de l'eau primaire est à $50^{\circ} \mathrm{C}$, à la phase sèche. À première vue, il n'y a pas d'interprétation logique mais ce fait doit être retenu dans la perspective de mesures ultérieures sur d'autres centrales.

Le chantier piscine n'a pu être traité qu'en présence de peu d'intervenants, d'où une faible mise en suspension des aérosols. La valeur élevée obtenue pour ${ }^{239-240} \mathrm{Pu}$ est à considérer comme un artéfact de mesure lié à des valeurs d'activité atmosphérique et surfacique pour ces isotopes proches de la limite de détection. Sur le chantier GV4 en phase humide, les facteurs de mise en suspension obtenus pour ${ }^{54} \mathrm{Mn}$ et ${ }^{56} \mathrm{Ni}$ sont élevés par rapport aux autres isotopes mais il existe une 


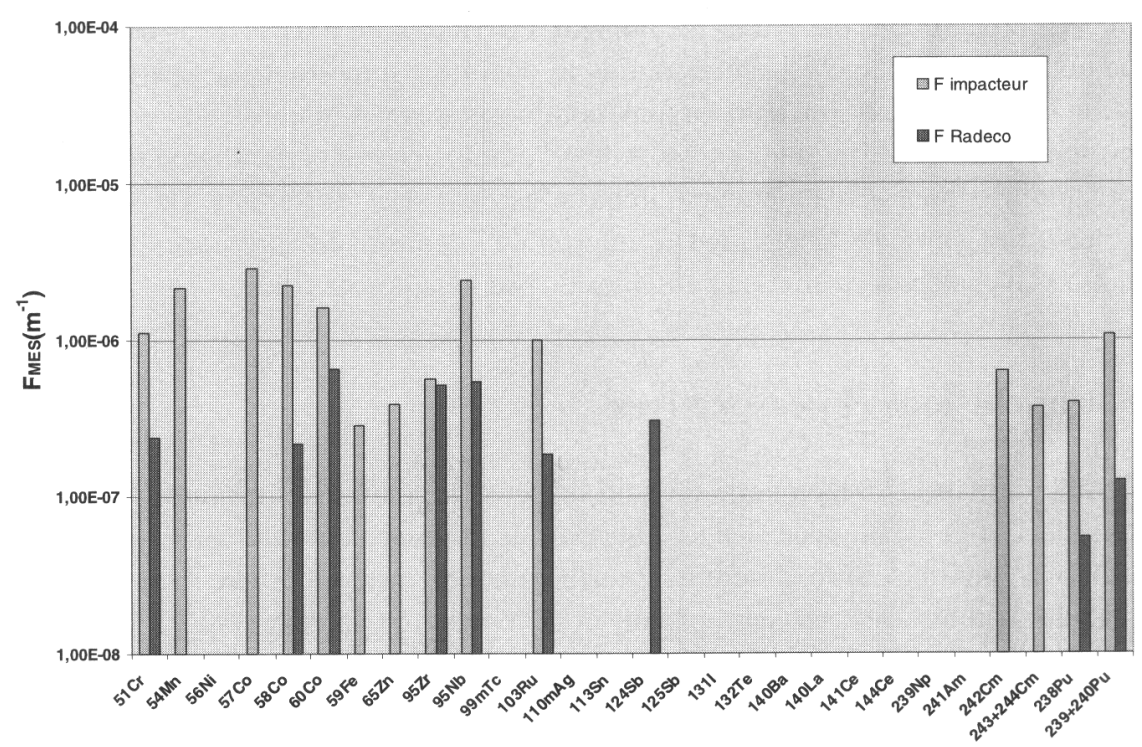

Figure 1 - Comparaison des facteurs de mise en suspension des aérosols dont les activités atmosphériques correspondantes sont mesurées avec l'aspirateur ou l'impacteur sur le chantier GV4 en phase sèche.

Comparison of atmospheric resuspension factor of aerosols on the intake and impactor of the vapor generator workplace in the dry phase.

forte incertitude sur la valeur de l'activité atmosphérique. La valeur du manganèse est cependant plus fiable. Le chantier en phase sèche correspond à des valeurs de $F_{\text {MES }}$ assez homogènes pour les différents isotopes.

Les mesures de ${ }^{90} \mathrm{Sr}$ réalisées sur les prélèvements atmosphériques sont toutes inférieures à la limite de détection. Aucun facteur de mise en suspension n'a donc pu être déterminé pour le strontium. Concernant les mesures sur les frottis, la valeur obtenue sur le sol de la piscine est particulièrement élevée par rapport à l'activité alpha et gamma de l'échantillon.

L'analyse de l'ensemble des activités volumiques des différents radio-isotopes sur le prélèvement atmosphérique réalisé sur le chantier GV4 sec à l'aide de l'impacteur Dekati PM-10, montre que les valeurs des émetteurs alpha sur l'impacteur sont plus significatives que sur le Radéco. À l'exception de ${ }^{241} \mathrm{Am}$, elles sont, pour la plupart, d'au moins 5 fois la limite de détection, ce qui donne des facteurs de mise en suspension des émetteurs alpha plus exploitables. On observe à partir du tableau III que les aérosols du prélèvement atmosphérique sont 
majoritairement des particules de diamètre inférieur à un micron. Plus le diamètre de coupure de l'étage est élevé, moins le dépôt est important. Les particules de taille supérieure à $10 \mu \mathrm{m}$ sont largement minoritaires. La majorité des isotopes est présente dans les différentes gammes de granulométrie, en particulier la répartition granulométrique des émetteurs alpha n'est pas très différente de celle des émetteurs gamma. Ceci peut s'interpréter comme la présence d'espèces radioactives déposées sur des particules de produits de corrosion.

\section{Conclusions et perspectives}

La détermination du facteur de mise en suspension déduite des mesures effectuées lors de l'arrêt de tranche 3 de Cattenom au premier semestre 2001 permet de proposer un inventaire d'activités surfaciques et atmosphériques sur les trois chantiers traités : pressuriseur, piscine et générateur de vapeur.

Il apparaît clairement que les prélèvements atmosphériques réalisés à l'aide de l'aspirateur Radéco conduisent à des activités alpha proches de la limite de détection et donc à des facteurs peu significatifs de mise en suspension des radioéléments émetteurs alpha.

L'impacteur donne des valeurs d'activités atmosphériques plus exploitables (au moins 5 fois la limite de détection) d'où des facteurs de mise en suspension plus homogènes entre les isotopes. L'impacteur permet en outre de donner des informations sur la taille des aérosols du prélèvement atmosphérique. Par exemple, sur le chantier générateur de vapeur en phase sèche, les particules ont un diamètre médian aérodynamique en activité proche de $1 \mu \mathrm{m}$. La répartition granulométrique des émetteurs alpha est assez homogène à celle des émetteurs gamma, ce qui est cohérent avec l'hypothèse de radioéléments émetteurs alpha et gamma présents sur les mêmes aérosols. L'impacteur n'a été utilisé que sur un chantier (GV4 sec) pour lequel on obtient une valeur moyenne du facteur de mise en suspension pour l'ensemble des isotopes égal à $1,2 \times 10^{-6} \mathrm{~m}^{-1}$. Pour ce chantier, pour les raisons mentionnées au paragraphe 5.2 le facteur de mise en suspension est en moyenne 5,5 fois plus élevé sur l'impacteur que sur le Radéco. Le chantier générateur de vapeur n'est pas enveloppe : la valeur moyenne maximale du facteur de mise en suspension mesurée à l'aide de l'aspirateur Radéco correspond au chantier pressuriseur en phase humide. Il semble donc difficile de préconiser une valeur unique pour le facteur de mise en suspension pour l'ensemble des chantiers. Il faut bien tenir compte qu'au stade actuel, l'étude n'a encore concerné qu'une seule installation industrielle avec les limites d'expérimentation qui découlent d'un travail sur le terrain. 
La comparaison avec les données de la littérature reste difficile. La majorité des valeurs de $\mathrm{F}_{\mathrm{MES}}$ recensées sont basées sur divers accidents radiologiques localisés ou des estimations faites en considérant la dissémination potentielle des produits de fission du réacteur de $2772 \mathrm{MW}$ thermiques de Three Miles Island, Brodsky (1980) avance une valeur moyenne $F_{\text {MES }}=1 \times 10^{-6} \mathrm{~m}^{-1}$. Dans sa synthèse, il la considère comme une valeur guide dans le cadre d'une protection radiologique usuelle mais la valeur $\mathrm{F}_{\mathrm{MES}}=1 \times 10^{-5} \mathrm{~m}^{-1}$ lui semble plutôt recommandable pour de longues durées d'exposition. Cette dernière valeur est alors considérée comme «As high as reasonably achievable » (AHARA). On peut donc considérer que nos résultats sont en accord raisonnable avec l'état de la question.

L'expérience de Cattenom est terminée, une campagne similaire de mesures a été réalisée sur le site de la centrale nucléaire de Golfech. Les données préliminaires de ces mesures sont en cours d'exploitation. Lors d'expérimentations ultérieures, il faudra nécessairement effectuer plus de prélèvements et surtout des prélèvements atmosphériques de longue durée permettant d'avoir accès notamment à la granulométrie des aérosols.

Remerciements. Les auteurs tiennent à remercier B. Rabu du CEA Cadarache, Dr J. Lallemand, Dr B. Le Guen, et A. Roupioz d'EDF pour les appuis techniques et logistiques qu'ils ont apporté pour la réalisation de ce travail.

\section{RÉFÉRENCES}

Alloul-Marmor L. (20)2) Ré-entraînement par écoulement d'air d'une contamination particulaire déposée sur une surface. Application au cas d'un «tas de poudre ». Thèse de l'université Paris-12.

Biasi L., De Los Reyes A., Reeks M.W., De Santi G.F. (2001) Use a simple model for the interpretation of experimental data on particle resuspension in turbulent flows, J. Aerosol Sci. 32, 1175-1200.

Brodsky A. (1980) Resuspension Factor and Probabilities of Intake of Material in Process (Ot is $10^{-6}$ a Magic Number in Health Physics), Health Phys. 39, 992-1000.

Cleaver J.W., Yates B. (1973) Mechanism of detachment of colloidal particles from a flat substrate in a turbulent flow, J. Coll. Interf. Sci. 44, 464-474.

Corino E.R., Brodkey R.S. (1969) A visual investigation of the wall region in turbulent flow, $J$. Fluid. Mech. 37, 1-30.

Décret (2003) Protection des travailleurs contre les dangers des rayonnements ionisants, Journal officiel de la république française, 2 avril 2003, décret $n^{\circ} 2003-196$ du 31 mars 2003-04-08.

DOE handbook (1994) Airborne release fractions/rates and respirable fractions for non reactor facilities. Volume 1, analyse of experiment data. Volume 2, Apendices. US Department of Energy Washington, DC.DOE-HDBK-3010-94.

NF-ISO 7503-1 (1998) Évaluation de la contamination de surface, partie I : émetteurs bêta (énergie beta maximale supérieure à $0,15 \mathrm{MeV}$ ) et émetteurs alpha.

Nicholson K.W. (1988) A review of particle resuspension, Atmos. Environ. 22, 2639-2651. 
Rabu B., Genin J.B., Leuthrot C., La Tiec C., Gimbert C. (2002) Compte-rendu de l'expérimentation du facteur de mise en suspension sur l'arrêt de tranche Cattenom 308, CEA, centre de Cadarache, note technique $\mathrm{DEC} / \mathrm{S} 3 \mathrm{C} / 01-101$.

Reeks M.W., Hall D. (2001) Kinetic models for particle suspension in turbulent flows: theory and measurement, J. Aerosol Sci. 32, 1-31.

Reeks M.W., Reeds J., Hall D. (1988) Kinetic models for particle suspension in turbulent flows: theory and measurement, J. Phys. D: Appl. Phys. 21, 574-589.

Renoux A., Boulaud D. (1998) Les Aérosols. Physique et métrologie. Édition Lavoisier, Technique et Documentation, Paris.

Sehmel G.A. (1980) Particle resuspension: a review, Environ. Int. 4, 107-127.

Witschger O. (1999) Mise en suspension de contamination particulaire radioactive, synthèse bibliographique, IPSN Rapport d'étude DPEA/SERAC/LPMA/99-13.

Ziskind G., Fichman M., Gutfinger C. (1995) Resuspension of particulates from surfaces to turbulent flows. Revicw and analysis, J. Aerosol Sci. 29, 613-644. 